\title{
The Absolute Certainty Principle (ACP) Revolutionizes Quantum Physics
}

\author{
Jean-Paul Auffray \\ Ex Courant Institute of Mathematical Sciences, New York University, New York, NY, USA \\ Email: jpauffray@yahoo.fr
}

How to cite this paper: Auffray, J.-P. (2016) The Absolute Certainty Principle (ACP) Revolutionizes Quantum Physics. Journal of Modern Physics, 7, 2341-2346.

http://dx.doi.org/10.4236/jmp.2016.716202

Received: November 14, 2016

Accepted: December 17, 2016

Published: December 20, 2016

Copyright $\odot 2016$ by author and Scientific Research Publishing Inc. This work is licensed under the Creative Commons Attribution International License (CC BY 4.0).

http://creativecommons.org/licenses/by/4.0/

\begin{abstract}
When the ubiquitous quantum, acting as an active principle, generates meteons in the System of the World, the Absolute Certainty Principle (ACP) regulates the characteristics of their motion. This newly uncovered law of Nature suggests that the cosmos is filled with an "aether", as Newton and others-even Einstein!_called it in their days, and explains quite simply why we stand erect vertically on the surface of the Earth and why the universe is in expansion.
\end{abstract}

\section{Keywords}

Heisenberg Uncertainty Principle, Quantum Meteorites, Absolute Certainty

Principle, Energy, Gravitation, Newton, Einstein, Universe Expansion

\section{Introduction}

Formulated in 1927 on the basis of an (ill-conceived) "thought experiment" [1], the "Heisenberg Uncertainty Principle" is a keystone of contemporary quantum physics (QP), and more precisely of Matrix Mechanics, also called Quantum Mechanics (QM). A recent publication by renowned Alexandria theoretician Mohamed S. El Naschie [2] brought into sharp focus an inherent characteristic of the Uncertainty Principle: as formulated in the context of QM it predicts-it requires-the existence in nature of "half quanta". This would seem to be incompatible with-and is indeed incompatible with-the widely held belief that the quantum-originally called Das Elementares Wirkungsquantum by its 1900 inventor, Max Planck-represents an entity-an element-which cannot be broken into parts smaller than itself. A dichotomy thus appears to exist—and indeed exists—at the very heart of QP [3]. We draw in the present note new conclusions from this unsatisfactory state of affairs. 


\section{QSM}

Our newly formulated Quantum Step Mechanics (QSM) [3] recognizes the presence in the Cosmos-in Nature-of a dimensionless Void, originally called the Zero Set by its inventor, Pr. M. S. El Naschie. The Zero set is said to contain "points" belonging in an Empty Set [4]. Busy constructing QSM step by step, we failed to notice right off the bat one incredibly significant detail affecting this scheme: the Empty set can properly be said to constitute... an "aether", as Newton and many others before and after him would spell it. This led us to examine in detail this possibility. We found that, when properly understood, QSM provides a (quantum) "mechanical model” of gravitation-a dream!! [5].

\section{The Problem with the Uncertainty Principle}

As we explain in detail in Heading 14 of [4], the Heisenberg Uncertainty Principle is rooted in the technical difficulties human observers encounter when they try to determine by means of light beams at the same time the position and the speed of particles-say electrons-in motion. It constitutes in effect a theory of measurement and does not express in earnest a true "law of nature". By contrast, following in Newton's footsteps (see Heading 5 of [5]), QSM assumes that an active principle-the Spirit and Will of God for Newton-the quantum for us today-intervenes permanently (continuously) in the affairs of the world, setting in motion randomly-selected points the Void-the Zero set-contains (Figure 1).

The proposal made in [5] to "explain" (to account for) gravitation in the context of the QSM scheme has one advantage: it works! Careful scrutiny by experts in the field will tell whether it constitutes a viable solution to this endemic problem.

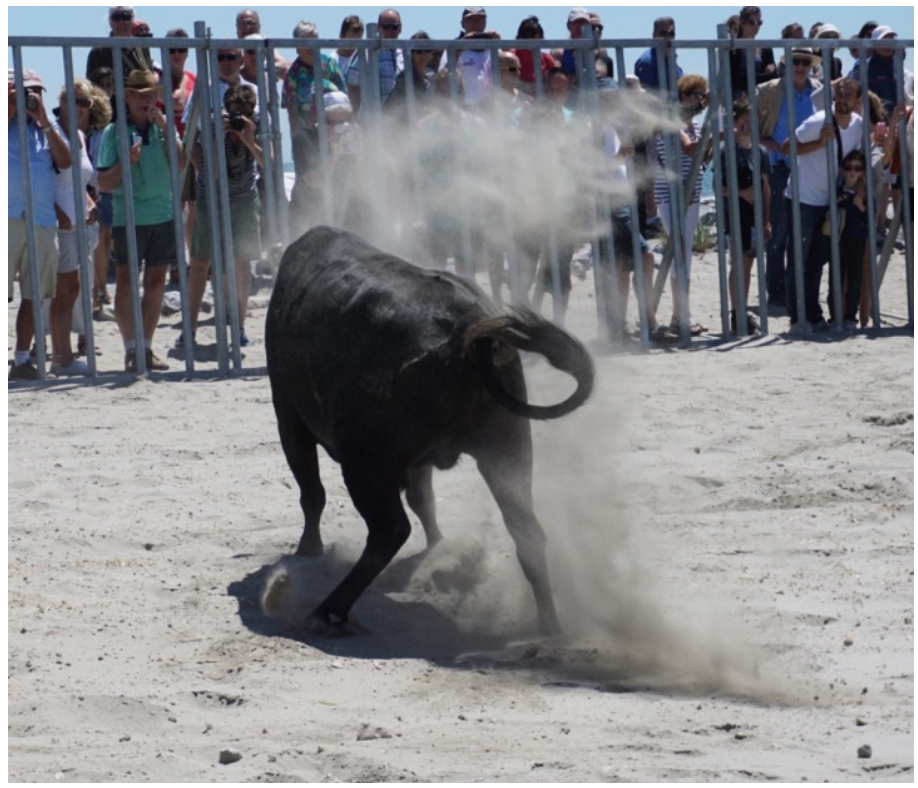

J. B. JPA private collection

Figure 1. An "active principle" setting "points" in motion in the Void. 
Faisant d'une pierre deux coups, as the French would say-killing two birds with one stone-QSM provides beyond gravitation a new insight in what might be the most disturbing question in modern physics: energy, what is it?

\section{Energy: What Is It?}

\subsection{We Do Not Know What Dark Energy Is}

In his celebrated Lectures on Physics the great Richard Feynman says it candidly: "It is important to realize that in physics today, we have no knowledge of what energy is", adding further on "It is a mathematical fiction (Feynman wrote 'an abstract thing')" [6].

In spite of this, to satisfy their insatiable need for energy, physicists around the world find it unabashedly wherever they want it to be: gravitational energy, kinetic energy, heat energy, elastic energy, electrical energy, chemical energy, radiant energy, nuclear energy, mass energy and... dark energy, said to constitute some $96 \%$ of the total energy present in the universe.

Where is that dark energy?

It is invisible, they say, this is why we call it dark (Figure 2).

\subsection{Dark Energy: The Phantom Is in the House}

Let the quantum, acting as an active principle, express itself by generating in the Void quantum meteorites-meteons by our terminology. For the main, four parameters determine the characteristics of their motion. Each meteon

- is driven by a momentum $p$,

- carries a (fractal) (wave) length $l$,

- and an electromagnetic pulse $E$

- for a time lapse of duration $d$.

There is more. A law of nature-the Absolute Certainty Principle (ACP) [3] - contrives these four parameters to satisfy with absolute certainty at least two quantum relations:

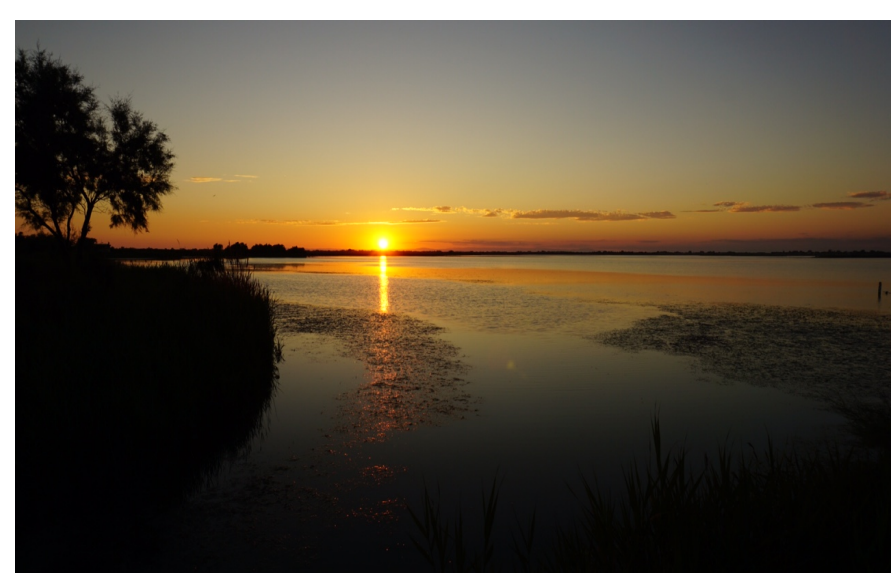

J. B. JPA private collection.

Figure 2. Dark energy-What is it? Where is it? 


$$
\begin{gathered}
h=l p \\
h=E d
\end{gathered}
$$

in which the so-called Planck constant $h$ measures the element of dynamical action (motion) which prevails in the System of the World. As for "energy"... We see that, whatever it is, it is generated continuously in the Void as a result of random quantum bursts- "xonic little bangs". In brief "energy", be it "ordinary" or "dark", is there, everywhere, all the time, around us (not "quantized"!): the phantom is in the house!

\section{Who's Afraid of Virginia Woolf?}

Our very use of the word "aether" in Heading 2 of the present note might be disconcerting to some of our readers who are used to regard the concept of an aether as being obsolete. And yet... some of the most outstanding and influential natural philosophers and physicists in the History of Science did use this word unabashedly in their writings in their days, for instance [our emphasizes throughout what follows]:

1) Isaac Newton who wrote in The Third Book of his Opticks (1718): "Is not this Medium [the aether] much rarer within the dense bodies of the Sun, stars, planets and comets, than in the empty celestial space between them?", to which he added: "And in passing from them to great distances, doth it not grow denser and denser perpetually, and thereby cause the gravity of those great bodies towards one another, and of their parts towards the bodies, everybody endeavouring to go from the denser parts of the Medium towards the rarer?' [7]. This would seem to be us speaking.

2) The ill-inspired inventor of the h-bar symbol [3], Nobel laureate Paul Dirac, who once expressed the thought that "the quantum vacuum may be the equivalent in modern physics of a particulate aether" [8].

3) And Einstein himself-yes, Einstein! - who once expressed this forceful belief, which will undoubtedly surprise some of his unconditional admirers: "According to the general theory of relativity space without aether is unthinkable" and he gave his own reasons to justify this conclusion: "For in such space there not only would be no propagation of light, but also no possibility of existence for standards of space and time (measuring-rods and clocks), nor therefore any space-time intervals in the physical sense." To which he added, thoughtfully: "But this aether may not be thought of as endowed with the quality characteristic of ponderable media, as consisting of parts which may be tracked through time. The idea of motion may not be applied to it." [9]

4) The great Dutch theoretician Hendrik Antoon Lorentz-the relativistic Lorentz transformation was named after him in June 1905 by its inventor, French mining engineer genius mathematician Henri Poincaré-believed to the end of his life in the presence of an aether in the universe. His only fundamental mistake was to assume this aether to be motionless. To us, made of quantum meteons in random motion, it is highly dynamical (Figure 3 ).

\section{An Unexpected Conclusion}

It all began in this journal on August 1, 2013, when we first expressed our ambitious 


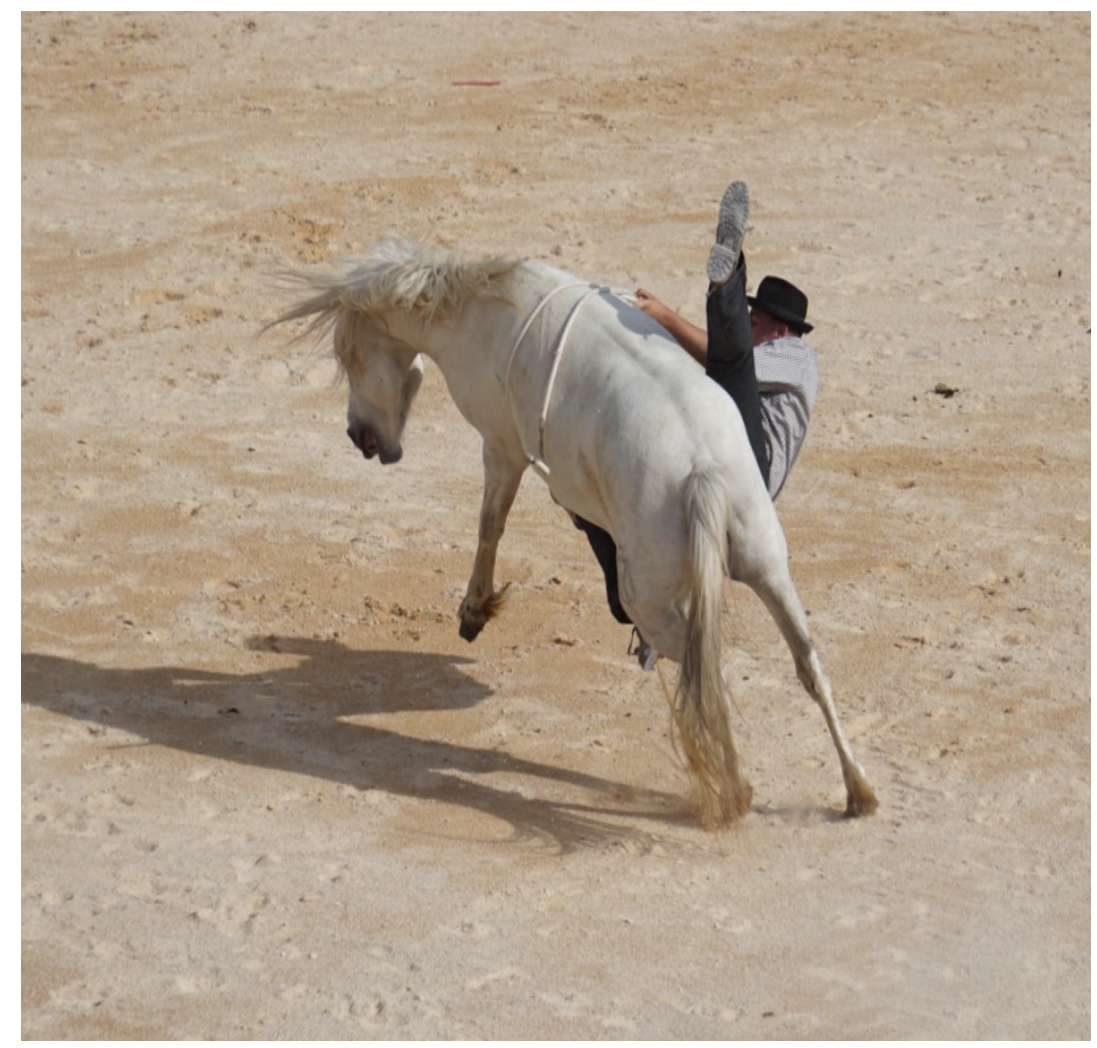

J. B. JPA private collection

Figure 3. Shaking off the uncertainty principle.

intention to try to convince (to incite) physicists at large around the world to stop using and abusing the words quantum and energy and to recognize instead that the symbol $h$ represents an element of dynamical action which must be treated respectfully accordingly [10]. We also urged physicists to start assessing properly in their historical contexts, Newton-no, he did not "explain" gravitation [5] — and Einstein-no, he did not "invent" $E=m c^{2}[12]$. And see what we ended up with three years later: silently ignoring our incitement to "reinvent quantum physics" [11], physicists at large around the world still use and abuse the words quantum and energy (as well as the names Newton and Einstein), and the expression dynamical action just about never appears in their writings. It is true that they had in this regard a prestigious and influential predecessor who managed to make the Planck constant disappear surreptitiously from his first major publication [12]. As a result, Quantum Physics remains in a bind. Our use of the word "aether" in the present note might provoke the seism hoped for. [And by the way, an aether permanently generated randomly in the cosmos by the ubiquitous quantum is a good candidate to be the fundamental cause of the strange observed expansion of the universe!].

In concluding this note, we feel entitled to proclaim with a degree of confidence-a degree of certainty! - that, in replacing the Heisenberg Uncertainty Principle, the ACP indeed revolutionizes Quantum Physics. A new era begins (Figure 4). 


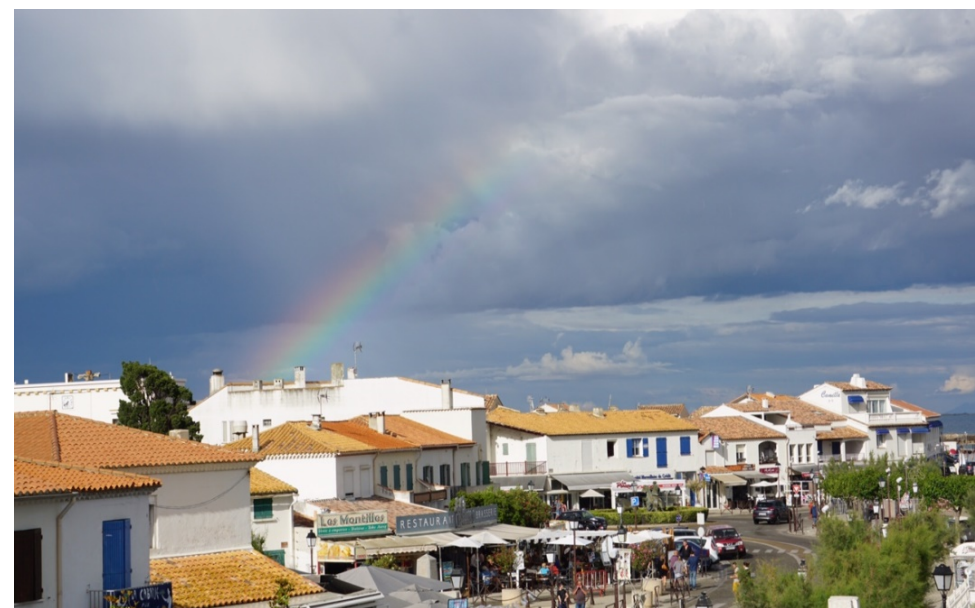

J. B. JPA private collection

Figure 4. ACP as a ray of hope in the sky of Quantum Physics.

\section{Acknowledgements}

We wish to express our deep appreciation to the JMP Editorial staff members for their valuable help and advice in properly preparing and formatting this note for online publication in their prestigious journal.

\section{References}

[1] Heisenberg, W. (1927) The Physical Content of Quantum Kinematics and Mechanics. In: Wheeler, J.A. and Zurek, W.H., Eds., Quantum Theory and Measurement, Princeton University Press, Princeton, 62-84. [Originally Published: Z. Phys., 1927, 43(3-4), 172-198].

[2] El Naschie, M.S. (2016) Journal of Modern Physics, 7, 1420-1428. https://doi.org/10.4236/jmp.2016.712129

[3] Auffray, J.-P. and El Naschie, M.S. (2016) Journal of Modern Physics, 7, 1949-1952. https://doi.org/10.4236/jmp.2016.714172

[4] Auffray, J.-P. (2015) Journal of Modern Physics, 6, 878-889. https://doi.org/10.4236/jmp.2015.67092

[5] Auffray, J.-P. (2016) Journal of Modern Physics, 7, 1702-1711. https://doi.org/10.4236/jmp.2016.713153

[6] Feynman, R.P., Leighton, R.B. and Sands, M. (2005) The Feynman Lectures on Physics: The Definitive and Extended Edition. 2nd Edition, Addison Wesley.

[7] Newton, I. (1718) The Third Book of Opticks, Query 21.

[8] Dirac, P. (1951) Nature, 168, 906. https://doi.org/10.1038/168906a0

[9] Einstein, A. (1920) Ether and the Theory of Relativity. Republished in Sidelights on Relativity. Methuen, London.

[10] Auffray, J.-P. (2013) Journal of Modern Physics, 4, 121-125. https://doi.org/10.4236/jmp.2013.48A011

[11] Auffray, J.-P. and El Naschie, M.S. (2016) Journal of Modern Physics, 7, 156-161. https://doi.org/10.4236/jmp.2016.71017

[12] Auffray, J.-P. (2015) Journal of Modern Physics, 6, 1478-1491. https://doi.org/10.4236/jmp.2015.611152 
Submit or recommend next manuscript to SCIRP and we will provide best service for you:

Accepting pre-submission inquiries through Email, Facebook, LinkedIn, Twitter, etc. A wide selection of journals (inclusive of 9 subjects, more than 200 journals)

Providing 24-hour high-quality service

User-friendly online submission system

Fair and swift peer-review system

Efficient typesetting and proofreading procedure

Display of the result of downloads and visits, as well as the number of cited articles

Maximum dissemination of your research work

Submit your manuscript at: http://papersubmission.scirp.org/

Or contact jmp@scirp.org 